\title{
CHAOTIC DYNAMICS OF DOMAIN WALLS IN MAGNETIC GARNET FILMS WITH IN-PLANE FIELDS
}

\author{
A. SUKIENNicki and R.A. Kosiński \\ Institute of Physics, Warsaw University of Technology \\ Koszykowa 75, 00-662 Warszawa, Poland
}

(Received January 30, 1995)

\begin{abstract}
Chaotic dynamics and routes to chaos of domain walls in magnetic bubble garnet materials in the presence of in-plane fields were investigated numerically using Slonczewski's equations of motion. Connection between the structure of the wall and the character of the attractor was found. The in-plane field can play a role of the factor controlling chaos.

PACS numbers: $05.45 .+b, 75.10 . H k, 75.60$. Ch
\end{abstract}

\section{Introduction}

Spatially extended nonlinear dynamical systems are recently extensively investigated. A good physical example of such a system is a domain wall in magnetic materials. In some conditions it exhibits an interesting chaotic behaviour [1-6]. Some years ago it was shown theoretically that for drive fields larger than the critical field in infinite material a structural transition occurs in the wall and a chaotic attractor appears in a properly chosen phase space [1]. On the other hand, recently, it has been found for finite thickness samples that, if the magnetic stray fields coming from surfaces of the material are taken into account in the calculations, a transition to chaos is realized via intermittency [5]. The so-called diffuse domain walls observed experimentally in bubble garnet materials by Vural and Humphrey [7] and analyzed numerically by Kosiński [8] can be regarded as a proof of the existence of chaotic motion of domain walls in such materials. On the other hand, external in-plane fields play an important role in various computer memory applications of domain walls and strongly influence their dynamics [9]. It is interesting therefore to investigate the influence of the external in-plane fields on the chaotic dynamics and route to chaos with the drive field treated as a control parameter. This is the subject of the present paper. 


\section{Equations of motion}

A domain wall in the uniaxial magnetic bubble garnet film is described by a pair of nonlinear partial differential equations, which have been derived by Slonczewski [10] from the Landau-Lifshitz equation with Gilbert's damping term included

$$
\begin{aligned}
& \frac{\dot{q}}{\Delta}=2 \pi M \gamma \sin 2 \psi-\frac{2 A \gamma}{M} \psi_{z z}+\frac{\gamma \pi}{2}\left[H_{x} \sin \psi-\left(H_{y}+H_{\mathrm{sy}}\right) \cos \psi\right]+\alpha \dot{\psi}, \\
& \dot{\psi}=\gamma H_{z}+\frac{2 \gamma A}{M \Delta} q_{z z}-\frac{\alpha}{\Delta} \dot{q},
\end{aligned}
$$

where the stray fields $H_{\text {sy }}$ from the surfaces of the film are taken in the form proposed by Hubert [11]

$$
H_{\mathrm{sy}}=4 M \ln \frac{h / 2-[z-2 \Delta \sinh (z / 2 \Delta) / \cosh (h / 4 \Delta)]}{h / 2+[z-2 \Delta \sinh (z / 2 \Delta) / \cosh (h / 4 \Delta)]} .
$$

Here, $A$ is the exchange constant, $\gamma$ is the gyromagnetic ratio, $4 \pi M$ is the saturation magnetization, $\alpha$ is the Gilbert damping constant, $\Delta=\sqrt{A / K}$ is the wall thickness parameter ( $K$ is the uniaxial perpendicular anisotropy constant), $H_{z}$ is the constant drive field applied perpendicularly to the film, $H_{x}$ and $H_{y}$ are the in-plane field components and $h$ is the film thickness. In Eqs. (1) and (2) $q(z, t)$ describes the local position of the Bloch surface of the wall and the azimuthal angle $\psi(z, t)$ describes the direction of the local magnetic moment of the wall with respect to $+0 x$ axis. The coordinate $z$ is perpendicular to the film and parallel to the easy axis of the uniaxial anisotropy of the film. A dot over a symbol denotes the time derivative and the subscript $z z-$ the second derivative with respect to the $z$ coordinate.

Equations of motion (1) and (2) were solved by means of a full implicit numerical scheme, which is described in Ref. [9]. Force-free boundary conditions were applied [10]. The initial conditions were $q(z, 0)=0, \psi(z, 0)=\psi_{s}(z)$, where $\psi_{\mathrm{s}}(z)$ is the static distribution resulting from the solution of Eqs. (1) and (2) with the time derivatives equal zero.

The material parameters were taken the same as in Ref. [5]. Namely, $A=$ $0.81 \times 10^{-7} \mathrm{erg} / \mathrm{cm}, 4 \pi M=140 \mathrm{G}, \gamma=1.75 \times 10^{7} \mathrm{~s}^{-1} \mathrm{Oe}^{-1}, \Delta=2.9 \times 10^{-6} \mathrm{~cm}$, $\alpha=0.156$ and $h=1.4 \times 10^{-4} \mathrm{~cm}$.

\section{Method of analysis}

To analyze the type of motion of the wall, the trajectory $\widetilde{q}(\widetilde{\psi})$ of the mid-point of the wall was calculated at each step of the integration procedure. The tilde denotes that the values of $\bar{q}$ and $\bar{\psi}$ averaged over the thickness of the film were subtracted from the instantaneous values of $q$ and $\psi$, respectively, because in this paper we are interested in the wall structure during the motion but not the overall translational motion of the wall.

Only asymptotical trajectories are analyzed, i.e. such for which all transients are ceased. 
Our discussion will concern only this range of drive fields in which a transition to chaos occurs (for small drives the domain wall motion is regular and will not be discussed here).

\section{Results and discussion}

In the case of the external in-plane field applied to the considered film a number of routes to chaos is found. Most common route to chaos is via intermittency - similar to the case of the zero external in-plane field [5]. For example, for $H_{y}=3$ Oe the upper bound of a periodic attractor is at $H_{z}=26.53 \mathrm{Oe}$ (Fig. 1), while for $H_{z}=26.54 \mathrm{Oe}$ intermittency appears (Fig. 2) and above $H_{z}=26.56 \mathrm{Oe}$ the chaotic attractor is observed (Fig. 3). Such a route to chaos occurs for small values of $H_{x}$ and $H_{y}$ (for $0<H_{x}<7 \mathrm{Oe}$ and for $0<H_{y}<4 \mathrm{Oe}$ ). Another route to chaos is found for $H_{y}=5 \mathrm{Oe}$, where the transition is realized via another periodic

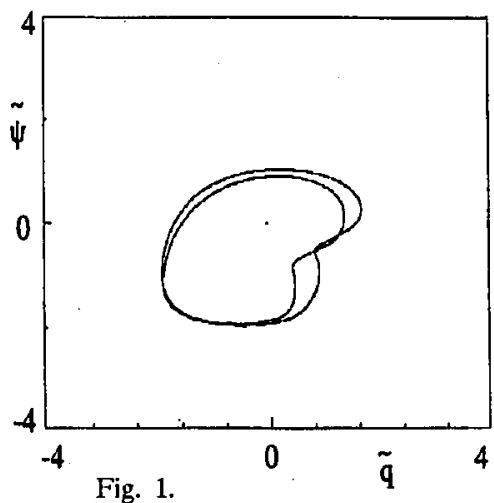

Fig. 1.

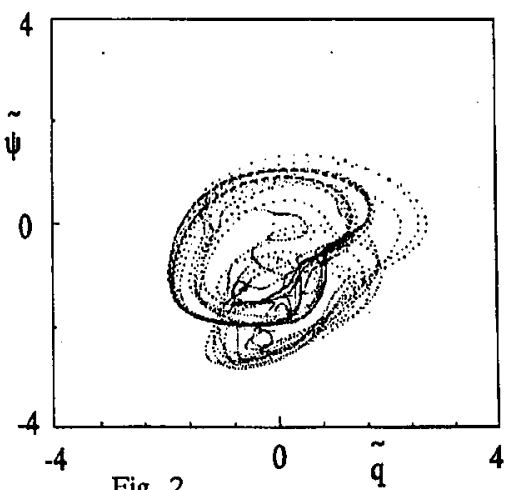

Fig. 2.

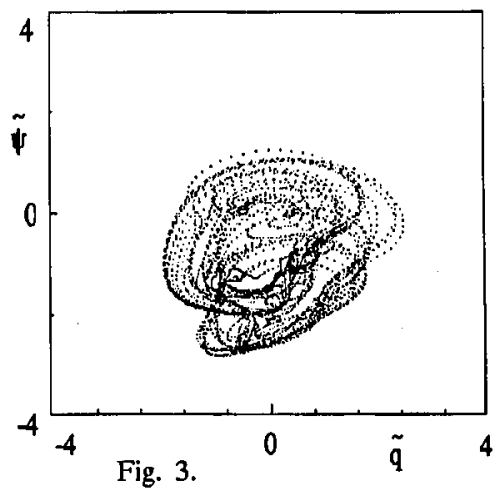

Fig. 1. Periodic attractor at $H_{y}=3 \mathrm{Oe}$ and $H_{z}=26.53 \mathrm{Oe}$

Fig. 2. Attractor for intermittency at $H_{y}=3 \mathrm{Oe}$ and $H_{z}=26.54$ Oe. Note the trace of the periodic attractor and otherwise the chaotic character.

Fig. 3. Chaotic attractor at $H_{y}=3$ Oe and $H_{z}=26.60$ Oe. 


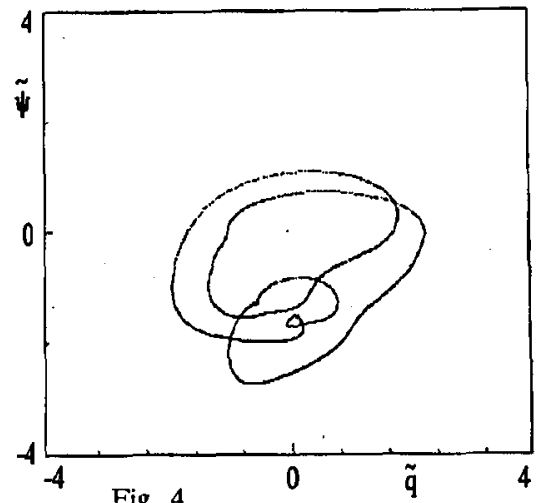

Fig. 4.

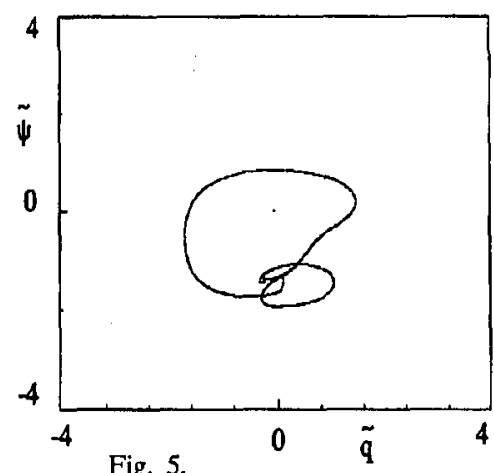

Fig. 5.
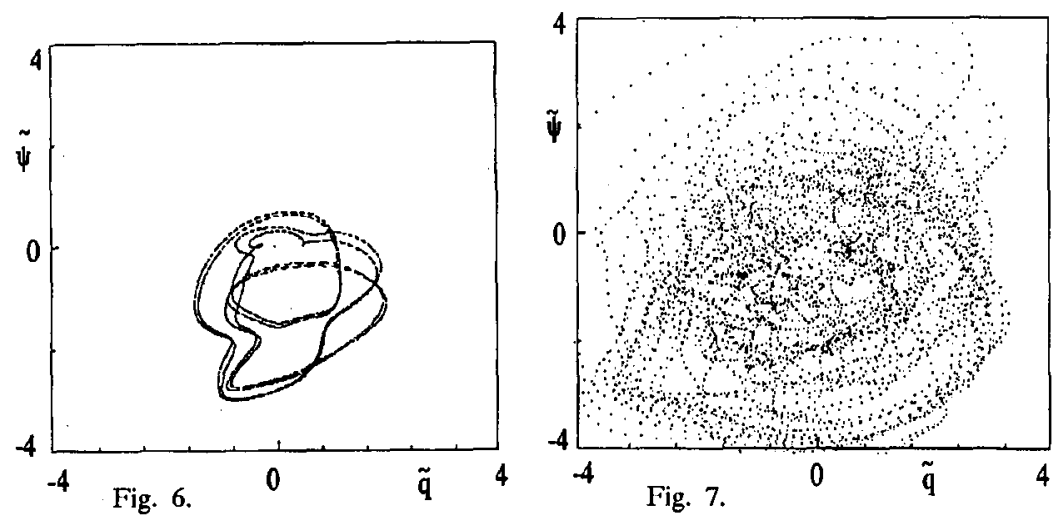

Fig. 4. Periodic attractor at $H_{y}=5 \mathrm{Oe}$ and $H_{z}=26.46 \mathrm{Oe}$.

Fig. 5. Periodic attractor at $H_{x}=20 \mathrm{Oe}$ and $H_{z}=25.38 \mathrm{Oe}$.

Fig. 6. Periodic attractor at $H_{x}=20 \mathrm{Oe}$ and $H_{z}=25.389 \mathrm{Oe}$.

Fig. 7. Chaotic attractor at $H_{x}=50 \mathrm{Oe}$ and $H_{z}=75 \mathrm{Oe}$.

attractor: at $H_{z}=26.45$ Oe the two-loop periodic attractor similar to that of Fig. 1 occurs, while for $H_{z}=26.46$ Oe the three-loop periodic attractor appears (Fig. 4) and it is stable up to $26.94 \mathrm{Oe}$, where the chaotic attractor appears. In this way the intermittency completely vanishes and the chaotic attractor is shifted to much larger values of $H_{z}$. It should be noted that the transition from the two-loop attractor to the three-loop attractor is realized by long lasting transient after which one of those attractors appears, depending on the value of the drive field. The third route to chaos has an intermediate character: e.g. for $H_{y}=4 \mathrm{Oe}$, above the range, where the two-loop attractor is observed - at $H_{z}=26.50 \mathrm{Oe}$, the intermittency appears at $26.51 \mathrm{Oe}$, the three-loop periodic attractor is at $26.53 \mathrm{Oe}$, and the chaotic attractor - at 26.55 Oe. In most frequent cases, when the route to chaos is via intermittency, the upper bound of the range in which periodic motion is observed as well as the lower bound of periodic motion decrease with an increase of the in-plane field. However, when the periodic attractor appears during the route to chaos, the chaotic attractor occurs at higher values of the in-plane field. 
Another route to chaos, where intermittency does not appear and the route to chaos is realized by a sequence of periodic attractors, was found for $H_{x}=20$ Oe. In this case a certain periodic attractor is observed at $H_{z}=25.38 \mathrm{Oe}$ (Fig. 5) while at 25.389 Oe the three-loop double splitted attractor occurs (Fig. 6), next the same attractor bifurcates further and finally the chaotic attractor is found at $26.60 \mathrm{Oe}$. It is interesting that for $H_{x}=-20 \mathrm{Oe}$, for $H_{y}=20 \mathrm{Oe}$ and for $H_{y}=-20 \mathrm{Oe}$ the standard route by intermittency appears.

For large values of in-plane fields chaos occurs at relatively high drive fields while for smaller values of $H_{z}$ another sequence of periodic attractors is obtained. For instance for $H_{x}=50$ Oe the transition to chaos is observed for drives $H_{z}$ about 51 Oe. The chaotic attractor found at $H_{z}=75 \mathrm{Oe}$ is shown in Fig. 7 .

It should be stressed that in the range of chaotic motion many periodic windows of different types and ranges of appearance were found. Most frequently, a three-loop periodic attractor (similar to that in Fig. 4) is present near the lower bound of the chaotic motion range:

In our computations the structure of the wall $\psi(z, l)$ was observed in order to connect it with the type of attractor. During the motion of the wall, some coherent spatial structures, the so-called horizontal Bloch lines; appear [10]. It was found that in the case of the small drives the motion of the horizontal Bloch lines with the angular span equal $\pi$ is regular and periodic, while an increase of the drive field and the in-plane field make the structure of the wall more complicated [9]. In particular the angular span of horizontal Bloch lines increases with an increase in the values of in-plane fields and their motion is highly irregular in time. For example, for $H_{y}=-50$ Oe the processes of the generation and annihilation of $4 \pi$ horizontal Bloch lines are observed. Regular or chaotic behaviour of the horizontal Bloch lines in the internal structures of the wall reflects itself in regular or chaotic type of motion and the level of complexity of attractor.

We found as an interesting result of our investigations that for some values of drive fields small changes of in-plane fields can cause the change of type of motion from chaotic to periodic one. It happens when the three-loop periodic attractor observed usually as a periodic window in the chaotic region shifts itself to such low values of drive fields that the intermittency vanishes completely. In these cases the chaotic motion is shifted to larger values of drive fields. This phenomenon can be treated as a kind of controlling chaos with the in-plane field as a controlling parameter. The role of the in-plane field is that it chooses some otherwise unstable orbit and makes it stable.

\section{Acknowledgments}

The work was supported by the State Committee for Scientific Research (Republic of Poland) under grant No. 223219102.

\section{References}

[1] J.J. Żebrowski, A. Sukiennicki, Acla Phys. Pol. A 72, 299 (1987).

[2] J.J. Żebrowski, A. Sukiennicki, Springer Proc. Phys. 23, 130 (1987). 
[3] R.A. Kosiński, J. Appl. Phys. 73, 320 (1992).

[4] R.A. Kosiński, IEEE Trans. Magn. 30, 1233 (1994).

[5] A. Sukiennicki, R.A. Kosiński, J. Magn. Magn. Mater. 129, 213 (1994).

[6] A. Sukiennicki, R.A. Kosiński, J.J. Żebrowski, Acta Phys. Pol. A 85, 791 (1994).

[7] K. Vural, F.B. Humphrey, J. Appl. Phys. 51, 549 (1980).

[8] R.A. Kosiński, Phys. Rev. B 50, 1241 (1994).

[9] R.A. Kosiński, J. Engemann, J. Magn. Magn. Mater. 50, 229 (1985).

[10] A.P. Malozemoff, J.C. Slonczewski, Magnetic Domain Walls in Bubble Materials, American Press, New York 1979.

[11] A. Hubert, J. Appl. Phys. 46, 2276 (1975). 